OPTIMAL CONVERGENCE OF THE ORIGINAL DG METHOD FOR THE TRANSPORT-REACTION EQUATION ON SPECIAL MESHES

By

\author{
Bernardo Cockburn \\ Bo Dong \\ and \\ Johnny Guzmán
}

IMA Preprint Series \# 2147

( December 2006)

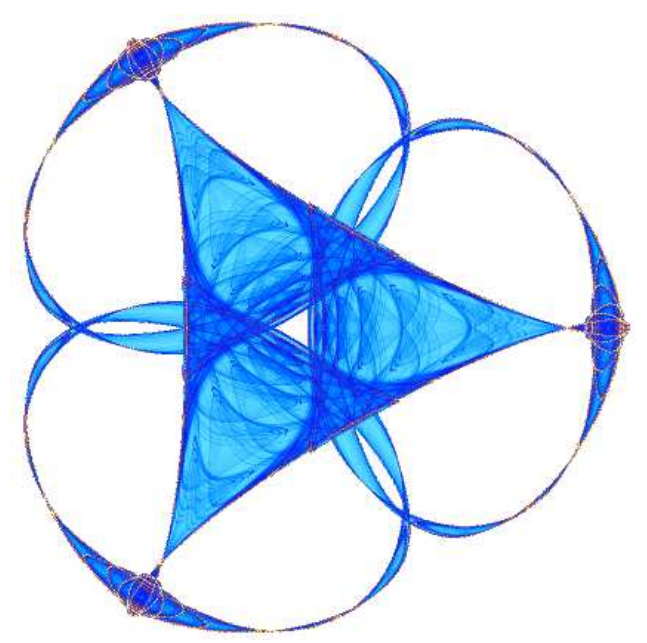

INSTITUTE FOR MATHEMATICS AND ITS APPLICATIONS

UNIVERSITY OF MINNESOTA

400 Lind Hall

207 Church Street S.E.

Minneapolis, Minnesota 55455-0436

Phone: 612/624-6066 Fax: 612/626-7370

URL: http://www.ima.umn.edu 


\title{
OPTIMAL CONVERGENCE OF THE ORIGINAL DG METHOD FOR THE TRANSPORT-REACTION EQUATION ON SPECIAL MESHES
}

\author{
BERNARDO COCKBURN *, BO DONG ${ }^{\dagger}$, AND JOHNNY GUZMÁN ‡
}

\begin{abstract}
We show that the approximation given by the original discontinuous Galerkin method for the transport-reaction equation in $d$ space dimensions is optimal provided the meshes are suitably chosen: the $L^{2}$-norm of the error is of order $k+1$ when the method uses polynomials of degree $k$. These meshes are not necessarily conforming and do not satisfy any uniformity condition; they are only required to be made of simplexes each of which has a unique outflow face. We also find a new, element-by-element postprocessing of the derivative in the direction of the flow which superconverges with order $k+1$.
\end{abstract}

Key words. discontinuous Galerkin methods, transport-reaction equation, error estimates

AMS subject classifications. $65 \mathrm{~N} 30,65 \mathrm{M} 60$

1. Introduction. We show that the original discontinuous Galerkin (DG) $[15$, 12], method can approximate in an optimal fashion the solution of the convectionreaction problem

$$
\begin{array}{rlrl}
\boldsymbol{\beta} \cdot \nabla u+c u & =f & \text { in } \Omega, \\
u & =g & & \text { on } \Gamma^{-} .
\end{array}
$$

Here $\Omega \subset R^{d}$ is a polyhedral domain, $\Gamma^{-}:=\{x \in \partial \Omega: \boldsymbol{\beta} \cdot \boldsymbol{n}(x)<0\}$, and $\boldsymbol{n}(x)$ is the outward unit normal at the point $x \in \partial \Omega$. The functions $f$ and $g$ are smooth, $\boldsymbol{\beta}$ is a non-zero constant unit vector and $c$ is a non-negative function in $L^{\infty}(\Omega)$. Indeed, if $u_{h}$ denotes the approximation given by the DG method using polynomials of degree $k$, we prove that, for a special class of triangulations $\Omega_{h}$, we have

$$
\left\|u-u_{h}\right\|_{L^{2}\left(\Omega_{h}\right)}+\left\|\mathrm{P}\left(\partial_{\boldsymbol{\beta}} u\right)-\partial_{\boldsymbol{\beta}, h} u_{h}\right\|_{L^{2}\left(\Omega_{h}\right)} \leq C|u|_{H^{k+1}\left(\Omega_{h}\right)} h^{k+1},
$$

where $\partial_{\boldsymbol{\beta}}:=\boldsymbol{\beta} \cdot \nabla, \mathrm{P}$ is the $L^{2}$-projection into the finite element space, and $\partial_{\boldsymbol{\beta}, h} u_{h}$ is an approximation to $\partial_{\boldsymbol{\beta}} u$ obtained by using an element-by-element postprocessing of $u_{h}$. Note that the above approximation result is optimal for the quantity $\left\|u-u_{h}\right\|_{L^{2}\left(\Omega_{h}\right)}$ in the order of convergence in $h$ as well as in the regularity of the exact solution; the estimate of the quantity $\left\|\mathrm{P}\left(\partial_{\boldsymbol{\beta}} u\right)-\partial_{\boldsymbol{\beta}, h} u_{h}\right\|_{L^{2}\left(\Omega_{h}\right)}$ is clearly a superconvergence result. This has to be contrasted with the estimate for general triangulations

$$
\left\|u-u_{h}\right\|_{L^{2}\left(\Omega_{h}\right)}+h^{1 / 2}\left\|\partial_{\boldsymbol{\beta}} u-\partial_{\boldsymbol{\beta}} u_{h}\right\|_{L^{2}\left(\Omega_{h}\right)} \leq C|u|_{H^{k+1}\left(\Omega_{h}\right)} h^{k+1 / 2},
$$

that follows from results obtained back in 1986 in [11].

The mechanisms that induce the loss of $h^{1 / 2}$ in the order of convergence of the $L^{2}$-norm of the error are not very well known yet. In 1988, it was shown [16] that, in the two-dimensional case, the estimate

$$
\left\|u-u_{h}\right\|_{L^{2}\left(\Omega_{h}\right)} \leq C|u|_{H^{k+2}\left(\Omega_{h}\right)} h^{k+1},
$$

*School of Mathematics, University of Minnesota, Vincent Hall, Minneapolis, MN 55455, USA, email: cockburn@math. umn. edu. Supported in part by the National Science Foundation (Grant DMS0411254) and by the University of Minnesota Supercomputing Institute.

${ }^{\dagger}$ School of Mathematics, University of Minnesota, Vincent Hall, Minneapolis, MN 55455, USA, email: bdong@math.umn.edu.

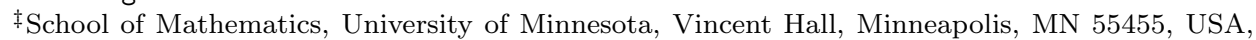
email: guzma033@umn.edu. Supported by an NSF Mathematical Science Postdoctoral Research Fellowship (DMS-0503050). 
holds for conforming triangulations obtained by using slabs of parallelograms divided into two triangles always in the same way. The triangles were chosen so that their sides are uniformly not aligned with the convection direction $\boldsymbol{\beta}$, that is, so that they satisfy what we could call the transversality condition

$$
\left|\boldsymbol{\beta} \cdot \boldsymbol{n}_{K}\right| \geq \gamma>0 \quad \text { on } \partial K, \quad \text { for all } K \in \Omega_{h},
$$

where $\boldsymbol{n}_{K}$ is the outward unit normal of the simplex $K$ and $\gamma$ is a fixed constant. In 1991, the rate of convergence of $h^{k+1 / 2}$ for $\left\|u-u_{h}\right\|_{L^{2}\left(\Omega_{h}\right)}$ was shown to be sharp in [13]; a rigorous proof was given for the case $k=0$ and convincing numerical evidence was shown for the case $k=1$, also in the two-dimensional case. As the reader might expect, in those numerical experiments, triangulations violating the transversality condition played a central role which lead the author to conjecture that this condition "may be a natural condition under which to seek improved estimates". However, a consequence of our main result is that improved estimates can be obtained even if the transversality condition is not satisfied in any triangle. Indeed, our improved estimates hold for triangulations $\Omega_{h}$ made of simplexes $K$ satisfying the simple flow conditions with respect to $\boldsymbol{\beta}$

Each simplex $K$ has a unique outflow face with respect to $\boldsymbol{\beta}, e_{K}^{+}$.

Each interior face $e_{K}^{+}$is included in an inflow face with respect to $\boldsymbol{\beta}$ of another simplex .

We say that the face $e$ of the simplex $K$ is an outflow (inflow) face with respect to $\boldsymbol{\beta}$ if $\left.\boldsymbol{\beta} \cdot \boldsymbol{n}_{K}\right|_{e}>(<) 0$. We say that a face is interior if it is not included in $\partial \Omega$. Note that the second condition allows the triangulation to be nonconforming. In two dimensions, this means that hanging nodes in a simplex $K$ are allowed provided they are not in its outflow edge; an example of such a triangulation is given in Fig. 1.1. Note that this triangulation is not uniform or translation invariant. In the Appendix, we show how to construct triangulations satisfying the flow conditions in any number of space dimensions.

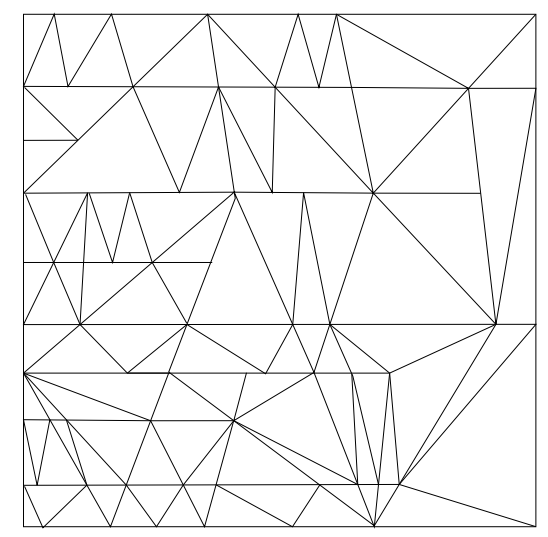

FIG. 1.1. A triangulation satisfying the flow conditions with $\boldsymbol{\beta}=(1,0)$. 
The main idea behind the devising of these meshes, and the associated analysis giving rise to optimal error estimates, is a suitable adaptation to the convectionreaction equation under consideration of the projection $(\boldsymbol{\Pi}, \mathbb{P})$ recently introduced for the analysis of superconvergent discontinuous Galerkin methods for second-order elliptic problems in [6]. The first component of such projection, $\boldsymbol{\Pi}$, was previously used in the analysis of the so-called minimal-dissipation LDG method for convectiondiffusion problems. Here, we use the second component, $\mathbb{P}$, to render the analysis of error in $u$ not only extremely simple and optimal but also capable of handling non-conforming meshes that are not uniform in any way.

Let us emphasize that the role of the special meshes for the construction of the approximation $\partial_{\boldsymbol{\beta}, h} u_{h}$ to the directional derivative $\partial_{\boldsymbol{\beta}} u$ is not essential for two reasons. The first is that such an approximation can be defined on any mesh of simplexes. The second is that, for the general meshes considered in [11], we can obtain the estimate

$$
\left\|\mathrm{P}\left(\partial_{\boldsymbol{\beta}} u\right)-\partial_{\boldsymbol{\beta}, h} u_{h}\right\|_{L^{2}\left(\Omega_{h}\right)} \leq C|u|_{H^{k+1}\left(\Omega_{h}\right)} h^{k+1 / 2}
$$

which shows that even in this case, $\partial_{\boldsymbol{\beta}, h} u_{h}$ is a better approximation than $\partial_{\boldsymbol{\beta}} u_{h}$. Postprocessings similar to the one giving rise to $\partial_{\boldsymbol{\beta}, h} u_{h}$ have been used before in the context of flow through porous media, [4], in the context of the Navier-Stokes equations, [7], and in the context of linear elastic incompressible materials, [8]. In all the above-mentioned references, they have been used to construct exactly divergencefree approximations to the velocity or exactly divergence-free stresses.

It is interesting to note that, when the triangulations $\Omega_{h}$ satisfy the flow conditions (1.3) with respect to $\boldsymbol{\beta}$ and with respect to $-\boldsymbol{\beta}$, we can obtain error estimates of the numerical trace $\widehat{u}_{h}$ in each outflow face $e$, namely,

$$
\left\|\mathrm{P}_{\partial} u-\widehat{u}_{h}\right\|_{H^{-s}(e)} \leq \mathrm{C}(c, s) h^{k+s+1}|u|_{H^{k+1}\left(\Omega_{e}\right)}
$$

for $s \in[0, k]$, where $\mathrm{P}_{\partial}$ is the $L^{2}$-projection into the space of polynomials of degree at most $k$ on each face, and $\Omega_{e}$ is a suitably chosen subset of $\Omega_{h}$. In particular, this implies that the average of $\widehat{u}_{h}$ on the face $e$ converges to the average of $u$ on that face with order $2 k+1$. This is the only result of this type in the current available scientific literature, to the knowledge of the authors.

Finally, we extend our results in two directions. First, we consider approximations that are polynomials of different degrees on different elements. With the condition that the degrees of polynomials are non-increasing in the direction of $\boldsymbol{\beta}$, we show that the estimates of approximations of $u$ and $\partial_{\boldsymbol{\beta}, h} u_{h}$ still hold. Then we consider the singularly perturbed problem in $\Omega \subset \mathbb{R}^{2}$

$$
\begin{aligned}
-\epsilon \Delta u+\boldsymbol{\beta} \cdot \nabla u+c u & =f & & \text { in } \Omega, \\
u & =g & & \text { on } \partial \Omega .
\end{aligned}
$$

where $0<\epsilon \ll 1$ is a constant and prove optimal local error estimates for $\epsilon \leq h^{2}$ on quasi-uniform meshes. We also show that internal numerical layers have width $\max \left(h, \epsilon^{1 / 2}\right)$ on triangulations $\Omega_{h}$ that satisfy the flow conditions (1.3) with respect to both $\boldsymbol{\beta}$ and $-\boldsymbol{\beta}$. This improves the best known result of $h^{1 / 2}$; see [10]. A similar result for the streamline-diffusion method is contained in [14].

The paper is organized as follows. In section 2 we state and prove our main results. In section 3, we extend our results to variable-degree versions of the method; we show the results for the singularly perturbed problem. We end in Section 4 with some concluding remarks. 


\section{The main results.}

2.1. The DG method. Suppose we have a family of triangulations $\left\{\Omega_{h}\right\}$ of $\Omega$ satisfying the flow conditions (1.3). To each triangulation $\Omega_{h}$, we associate the number $h=\sup _{K \in \Omega_{h}} h_{K}$, where $h_{K}=\operatorname{diam}(K)$, and the finite-dimensional space $V_{h}^{k}$ which is composed of functions that are polynomials of degree at most $k$ on each simplex $K \in \Omega_{h}$.

The DG approximation $u_{h} \in V_{h}^{k}$ of the solution of (1.1) solves

$$
B\left(u_{h}, v_{h}\right)=\left(f, v_{h}\right)_{\partial \Omega_{h}}-\left\langle g, v_{h} \boldsymbol{\beta} \cdot \boldsymbol{n}\right\rangle_{\Gamma^{-}}, \quad \text { for all } v_{h} \in V_{h}^{k},
$$

where

$$
B(w, v)=-\left(w, \partial_{\boldsymbol{\beta}} v\right)_{\Omega_{h}}+\langle\widehat{w}, v \boldsymbol{\beta} \cdot \boldsymbol{n}\rangle_{\partial \Omega_{h} \backslash \Gamma^{-}}+(c w, v)_{\Omega_{h}},
$$

for any $w, v$ in $H^{1}\left(\Omega_{h}\right)$. Notice that we only need to define the numerical trace $\widehat{v}$ on faces that are not parallel to the direction $\boldsymbol{\beta}$ and do not belong to the inflow part of the boundary $\Gamma^{-}$. Therefore, the numerical trace of a function $v$ on a simplex $K$ for such faces $e$ is given by

$$
\widehat{v}:=v^{-},
$$

where $v^{ \pm}(z)=\lim _{\delta \downarrow 0} v(z \pm \delta \boldsymbol{\beta})$ where $z \in e$. We are using the notation

$$
\begin{aligned}
(\boldsymbol{\sigma}, \boldsymbol{v})_{\Omega_{h}} & :=\sum_{K \in \Omega_{h}} \int_{K} \boldsymbol{\sigma}(x) \cdot \boldsymbol{v}(x) d x, \quad(\zeta, \omega)_{\Omega_{h}}:=\sum_{K \in \Omega_{h}} \int_{K} \zeta(x) \omega(x) d x, \\
\langle\zeta, \boldsymbol{v} \cdot \boldsymbol{n}\rangle_{\partial \Omega_{h}} & :=\sum_{K \in \Omega_{h}} \int_{\partial K} \zeta(\gamma) \boldsymbol{v}(\gamma) \cdot \boldsymbol{n} d \gamma,
\end{aligned}
$$

for any functions $\boldsymbol{\sigma}, \boldsymbol{v}$ in $\boldsymbol{H}^{1}\left(\Omega_{h}\right):=\left[H^{1}\left(\Omega_{h}\right)\right]^{d}$ and $\zeta, \omega$ in $H^{1}\left(\Omega_{h}\right)$. The outward normal unit vector to $\partial K$ is denoted by $\boldsymbol{n}$.

Notice that the exact solution $u$ of (1.1) also satisfies the weak formulation (2.1), so we have the error equation

$$
B\left(u-u_{h}, v_{h}\right)=0 \quad \text { for all } v_{h} \in V_{h}^{k} .
$$

2.2. The approximation of $u$. To state our result on the approximation of $u$, we need to introduce a special projection, $\mathbb{P}$, defined on triangulations $\Omega_{h}$ satisfying the flow condition (1.3a). The function $\mathbb{P} u \in V_{h}^{k}$ restricted to $K \in \Omega_{h}$ is given by

$$
\begin{aligned}
(\mathbb{P} u-u, v)_{K} & =0, & & \text { for all } v \in \mathcal{P}^{k-1}(K) \\
\langle\mathbb{P} u-u, w\rangle_{e_{K}^{+}} & =0, & & \text { for all } w \in \mathcal{P}^{k}\left(e_{K}^{+}\right),
\end{aligned}
$$

The following lemma was proved in [6].

LEMma 2.1. If the triangulation $\Omega_{h}$ satisfies the flow condition (1.3a), the projection $\mathbb{P}$ given by (2.3) is well defined. Moreover, on each simplex $K \in \Omega_{h}$ we have

$$
\|\mathbb{P} u-u\|_{L^{2}(K)} \leq C h^{k+1}|u|_{H^{k+1}(K)},
$$

where $C$ only depends on $k$ and the shape regularity of the simplex $K$.

Now we can state our first error estimate. 
THEOREM 2.2. If $\Omega_{h}$ satisfies the flow conditions (1.3), the error between $u_{h}$ given by the discontinuous Galerkin method (2.1) and the exact solution $u$ of the equations (1.1) is bounded as follows:

$$
\left\|\mathbb{P} u-u_{h}\right\|_{L^{2}\left(\Omega_{h}\right)} \leq C\|c(u-\mathbb{P} u)\|_{L^{2}\left(\Omega_{h}\right)} .
$$

In particular, if $c \equiv 0$ then

$$
u_{h}=\mathbb{P} u \text {. }
$$

Note that, after a straightforward application of the triangle inequality, we get

$$
\left\|u-u_{h}\right\|_{L^{2}\left(\Omega_{h}\right)} \leq\|u-\mathbb{P} u\|_{L^{2}\left(\Omega_{h}\right)}+C\|c(u-\mathbb{P} u)\|_{L^{2}\left(\Omega_{h}\right)},
$$

and hence, by the approximation property of the projection $\mathbb{P},(2.4)$, we have that

$$
\left\|u-u_{h}\right\|_{L^{2}\left(\Omega_{h}\right)} \leq C|u|_{H^{k+1}\left(\Omega_{h}\right)} h^{k+1},
$$

whenever $u \in H^{k+1}\left(\Omega_{h}\right)$, as claimed in the Introduction.

Proof. Set $\mathbb{E}=u_{h}-\mathbb{P} u$. By the error equation (2.2), we have that for all $v \in V_{h}^{k}$,

$$
B(\mathbb{E}, v)=B(u-\mathbb{P} u, v)=\sum_{i=1}^{3} T_{i}
$$

where, by definition of the bilinear form $B(\cdot, \cdot),(2.1 \mathrm{~b})$,

$$
\begin{aligned}
& T_{1}=-\left(u-\mathbb{P} u, \partial_{\boldsymbol{\beta}} v\right)_{\Omega_{h}}, \\
& T_{2}=\langle\widehat{u}-\widehat{\mathbb{P} u}, v \boldsymbol{\beta} \cdot \boldsymbol{n}\rangle_{\partial \Omega_{h} \backslash \Gamma^{-}}, \\
& T_{3}=(c(u-\mathbb{P} u), v)_{\Omega_{h}} .
\end{aligned}
$$

Now, by the definition of the projection $\mathbb{P},(2.3 \mathrm{a})$,

$$
T_{1}=0 \text {. }
$$

Moreover, by the definition of the numerical trace $\widehat{\mathbb{P}} u,(2.1 \mathrm{c})$,

$$
\begin{aligned}
T_{2} & =\langle u-\widehat{\mathbb{P} u}, v \boldsymbol{\beta} \cdot \boldsymbol{n}\rangle_{\partial \Omega_{h} \backslash \Gamma^{-}} \\
& =\sum_{K \in \Omega_{h}}\left\langle u-\mathbb{P} u,\left(v^{-}-v^{+}\right) \boldsymbol{\beta} \cdot \boldsymbol{n}\right\rangle_{e_{K}^{+}} \\
& =0
\end{aligned}
$$

by the definition of the projection $\mathbb{P},(2.3 \mathrm{~b})$, and by the fact that, by the second flow condition $(1.3 \mathrm{~b}),\left.\left(v^{-}-v^{+}\right)\right|_{e_{K}^{+}} \in \mathcal{P}^{k}\left(e_{K}^{+}\right)$. Therefore, we have that

$$
B(\mathbb{E}, v)=(c(u-\mathbb{P} u), v)_{\Omega_{h}} \text { for all } v \in V_{h}^{k},
$$

and, by the stability result of Theorem 2.1 in [11], we obtain

$$
\|\mathbb{E}\|_{L^{2}\left(\Omega_{h}\right)} \leq C\|c(u-\mathbb{P} u)\|_{L^{2}\left(\Omega_{h}\right)},
$$

for $h$ sufficiently small. This completes the proof. 
2.3. Post-processing: The approximation to $\partial_{\boldsymbol{\beta}} u$. Next we show how to post-process $u_{h}$ in order to get a superconvergent approximation of $\partial_{\boldsymbol{\beta}} u$. To this end, for each simplex $K$ we define $\boldsymbol{q}_{h} \in \mathcal{P}^{k}(K) \oplus \boldsymbol{x} \mathcal{P}^{k}(K)$ to be the solution of

$$
\begin{aligned}
& \left(\boldsymbol{q}_{h}-\boldsymbol{\beta} u_{h}, \boldsymbol{v}\right)_{K}=0, \quad \text { for all } \boldsymbol{v} \in \mathcal{P}^{k-1}(K) \\
& \left\langle\left(\boldsymbol{q}_{h}-\boldsymbol{\beta} \lambda_{h}\right) \cdot \boldsymbol{n}, w\right\rangle_{e}=0, \quad \text { for all } w \in \mathcal{P}^{k}(e) \text {, for all faces } e \text { of } K \text {, }
\end{aligned}
$$

where $\lambda_{h}=\mathrm{P}_{\partial} g$ on $\Gamma^{-}$and $\lambda_{h}=\widehat{u}_{h}$ otherwise. The existence and uniqueness of $\boldsymbol{q}_{h}$ is well known; see, for example, [5]. We then define

$$
\partial_{\boldsymbol{\beta}, h} u_{h}:=\nabla \cdot \boldsymbol{q}_{h} \quad \text { in } \Omega_{h}
$$

We can now state the error estimate between $\partial_{\boldsymbol{\beta}, h} u_{h}$ and $\partial_{\boldsymbol{\beta}} u$.

THEOREM 2.3. If $\Omega_{h}$ is an arbitrary triangulation of $\Omega$, then

$$
\left\|\partial_{\boldsymbol{\beta}, h} u_{h}-\mathrm{P}\left(\partial_{\boldsymbol{\beta}} u\right)\right\|_{L^{2}\left(\Omega_{h}\right)} \leq C\left\|c\left(u-u_{h}\right)\right\|_{L^{2}\left(\Omega_{h}\right)} .
$$

In particular, if $c \equiv 0$ then

$$
\partial_{\boldsymbol{\beta}, h} u_{h}=\mathrm{P}\left(\partial_{\boldsymbol{\beta}} u\right) .
$$

Here $\mathrm{P}$ is the $L^{2}$-projection into $V_{h}^{k}$.

We can thus see that, if we use the estimates for arbitrary triangulations $\Omega_{h}$ obtained in [11], we obtain

$$
\left\|\mathrm{P}\left(\partial_{\boldsymbol{\beta}} u\right)-\partial_{\boldsymbol{\beta}, h} u_{h}\right\|_{L^{2}\left(\Omega_{h}\right)} \leq C|u|_{H^{k+1}\left(\Omega_{h}\right)} h^{k+1 / 2}
$$

and if, we assume that $\Omega_{h}$ satisfies the flow conditions (1.3), we obtain, by Theorem 2.2 ,

$$
\left\|\mathrm{P}\left(\partial_{\boldsymbol{\beta}} u\right)-\partial_{\boldsymbol{\beta}, h} u_{h}\right\|_{L^{2}\left(\Omega_{h}\right)} \leq C|u|_{H^{k+1}\left(\Omega_{h}\right)} h^{k+1},
$$

as claimed in the Introduction. This implies that, if $u$ is smooth enough, $\| \partial_{\boldsymbol{\beta}} u-$ $\partial_{\boldsymbol{\beta}, h} u_{h} \|_{L^{2}\left(\Omega_{h}\right)}$ is of order $h^{k+1 / 2}$ for arbitrary meshes and of order $h^{k+1}$ for the special meshes under consideration.

Proof. For $v \in V_{h}^{k}$ we have

$$
\begin{aligned}
\left(\partial_{\boldsymbol{\beta}, h} u_{h}-\mathrm{P}\left(\partial_{\boldsymbol{\beta}} u\right), v\right)_{\Omega_{h}} & =\left(\nabla \cdot \boldsymbol{q}_{h}-\partial_{\boldsymbol{\beta}} u, v\right)_{\Omega_{h}} \\
& =-\left(\boldsymbol{q}_{h}, \nabla v\right)_{\Omega_{h}}+\left\langle\boldsymbol{q}_{h} \cdot \boldsymbol{n}, v\right\rangle_{\partial \Omega_{h}}-\left(\partial_{\boldsymbol{\beta}} u, v\right)_{\Omega_{h}} \\
& =-\left(\boldsymbol{q}_{h}, \nabla v\right)_{\Omega_{h}}+\left\langle\boldsymbol{q}_{h} \cdot \boldsymbol{n}, v\right\rangle_{\partial \Omega_{h}}-(f, v)_{\Omega_{h}}+(c u, v)_{\Omega_{h}},
\end{aligned}
$$

by the definition of the exact solution $u$ of (1.1a). By the definition of $\boldsymbol{q}_{h},(2.5)$,

$$
\begin{aligned}
\left(\partial_{\boldsymbol{\beta}, h} u_{h}-\mathrm{P}\left(\partial_{\boldsymbol{\beta}} u\right), v\right)_{\Omega_{h}}= & -\left(u_{h}, \partial_{\boldsymbol{\beta}} v\right)_{\Omega_{h}}+\left\langle\widehat{u}_{h}, v \boldsymbol{\beta} \cdot \boldsymbol{n}\right\rangle_{\Omega_{h} \backslash \Gamma^{-}}+\langle g, v \boldsymbol{\beta} \cdot \boldsymbol{n}\rangle_{\Gamma^{-}} \\
& -(f, v)_{\Omega_{h}}+(c u, v)_{\Omega_{h}} \\
= & \left(c\left(u-u_{h}\right), v\right)_{\Omega_{h}},
\end{aligned}
$$

by the definition of the approximate solution $u_{h},(2.1)$. The proof is complete once we take $v=\partial_{\boldsymbol{\beta}, h} u_{h}-\mathrm{P}\left(\partial_{\boldsymbol{\beta}} u\right)$. 
2.4. The approximation of the numerical trace $\widehat{u}_{h}$ on outflow faces. To state the approximation result, let us introduce some notation. We are going to use the following negative-order norm

$$
\|\gamma\|_{H^{-s}(e)}:=\sup _{\varphi \in \mathcal{C}^{\infty}(e)} \frac{\langle\gamma, \varphi\rangle_{e}}{\|\varphi\|_{H^{s}(e)}},
$$

where $e$ is the outflow face of the simplex $K \in \Omega_{h}$. To this face, we associate the subset of $\Omega_{h}$ defined by

$\Omega_{e}:=\left\{K \in \Omega_{h}: \forall x \in K\right.$, there is $x_{0} \in e$ and $t \geq 0$, such that $\left.x=x_{0}-\boldsymbol{\beta} t\right\}$.

THEOREM 2.4. If $\Omega_{h}$ is a triangulation of $\Omega$ satisfying the flow conditions (1.3), with respect to $\boldsymbol{\beta}$ and $-\boldsymbol{\beta}$. Then on any outflow face $e$

$$
\left\|\mathrm{P}_{\partial} u-\widehat{u}_{h}\right\|_{H^{-s}(e)} \leq \mathrm{C}(c, s) h^{k+s+1}|u|_{H^{k+1}\left(\Omega_{e}\right)},
$$

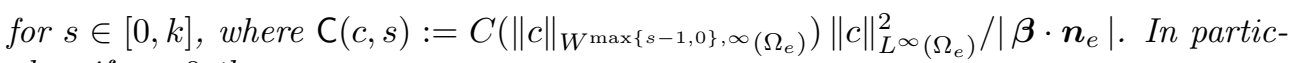
ular, if $c \equiv 0$ then

$$
\mathrm{P}_{\partial} u=\widehat{u}_{h} .
$$

Note that the condition of the triangulation $\Omega_{h}$ implies that each simplex $K$ has exactly one outflow face $e_{K}^{+}$and one inflow face $e_{K}^{-}$. It also implies that the outflow face $e_{K}^{+}$lies on $\partial \Omega \backslash \Gamma^{-}$or coincides with an inflow face, and that $e_{K}^{-}$lies on $\Gamma^{-}$or coincides with an outflow face.

This result implies, in particular, that the average of the numerical trace $\widehat{u}_{h}$ on each outflow face $e$ converges to the average of the exact solution $u$ on that face with order $2 k+1$ for general $c$ and with order $2 k+2$ in the case $c=0$. To see this, let us begin by noting that if $\Upsilon \in H^{k+1}(e)$, we have that

$$
\begin{aligned}
\left\langle\widehat{u}_{h}-u, \Upsilon\right\rangle_{e} & =\left\langle\widehat{u}_{h}-\mathrm{P}_{\partial} u, \Upsilon\right\rangle_{e}+\left\langle\mathrm{P}_{\partial} u-u, \Upsilon\right\rangle_{e} \\
& =\left\langle\widehat{u}_{h}-\mathrm{P}_{\partial} u, \Upsilon\right\rangle_{e}+\left\langle\mathrm{P}_{\partial} u-u, \Upsilon-\mathrm{P}_{\partial} \Upsilon\right\rangle_{e}
\end{aligned}
$$

and so

$$
\left|\left\langle\widehat{u}_{h}-u, \Upsilon\right\rangle_{e}\right| \leq C h^{2 k+1}
$$

where

$$
C=\mathrm{C}(c, s)|u|_{H^{k+1}\left(\Omega_{e}\right)}\|\Upsilon\|_{H^{k+1}(e)}+C h|u|_{H^{k+1}(e)}|\Upsilon|_{H^{k+1}(e)} .
$$

The claim follows by simply taking $\Upsilon=1$.

Let us emphasize that the order of convergence of the approximation of linear functionals of the form $\langle u, \Upsilon\rangle_{\partial \Omega \backslash \Gamma^{-}}$can be proven to be of order $2 k+1$; see [1] for the case $c=0$ and $\Upsilon=1$. This result holds for functions $\Upsilon$ that are independent of the mesh; as a consequence, $\Upsilon$ cannot be taken to have support in a single element face. To the knowledge of the authors, Theorem 2.4 is the only result that allows this.

Next, we give a proof of the result. To do that, we need to introduce some notation. A glance to the definition of the negative-order norm $\|\cdot\|_{H^{-s}\left(\Omega_{e}\right)},(2.6)$, 
suggests that, to estimate $\left\|\mathrm{P}_{\partial} u-\widehat{u}_{h}\right\|_{H^{-s}(e)}$, we need to introduce the corresponding dual problem, namely,

$$
\begin{aligned}
-\boldsymbol{\beta} \cdot \nabla \psi+c \psi & =0 \quad \text { in } \Omega_{e}, \\
\psi & =\varphi / \boldsymbol{\beta} \cdot \boldsymbol{n} \text { on } e,
\end{aligned}
$$

whose exact solution is

$$
\psi(x(t))=\varphi\left(x_{0}\right) e^{-\int_{0}^{t} c(x(\tau)) d \tau} / \boldsymbol{\beta} \cdot \boldsymbol{n} .
$$

where $x(t)=x_{0}-\boldsymbol{\beta} t$. Hence, if we assume that $c \in W^{\max \{s-1,0\}, \infty}\left(\Omega_{e}\right)$ and that $\psi \in H^{s}\left(\Omega_{e}\right)$ for some $s \geq 0$, then we have

$$
|\psi|_{H^{s}\left(\Omega_{e}\right)} \leq C\|\varphi\|_{H^{s}(e)} / \boldsymbol{\beta} \cdot \boldsymbol{n}
$$

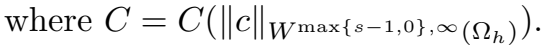

We are going to use two auxiliary projections. We set $\mathbb{P}^{+}:=\mathbb{P}$ which is defined in $(2.3)$, and let $\mathbb{P}^{-}$be the projection which satisfies $(2.3 \mathrm{a})$ and imposes $(2.3 \mathrm{~b})$ on $e_{K}^{-}$instead of $e_{K}^{+}$. Finally, we note that, by using the projections $\mathbb{P}^{+}$and $\mathrm{P}_{\partial}$, we can rewrite the error equation $(2.2)$ as

$$
-\left(\mathbb{P}^{+} u-u_{h}, \partial_{\boldsymbol{\beta}} v\right)_{\Omega_{e}}+\left\langle\mathrm{P}_{\partial} u-\widehat{u}_{h}, v \boldsymbol{\beta} \cdot \boldsymbol{n}\right\rangle_{\partial \Omega_{e} \backslash \Gamma^{-}}+\left(c\left(u-u_{h}\right), v\right)_{\Omega_{e}}=0 .
$$

We are now ready to prove Theorem 2.4.

Proof. To prove this result, we begin by noting that

$$
\left\langle\mathrm{P}_{\partial} u-\widehat{u}_{h}, \varphi\right\rangle_{e}=\left\langle\mathrm{P}_{\partial} u-\widehat{u}_{h}, \psi \boldsymbol{\beta} \cdot \boldsymbol{n}\right\rangle_{e}
$$

by the boundary condition of the dual problem, (2.7b), and that

$$
\left\langle\mathrm{P}_{\partial} u-\widehat{u}_{h}, \varphi\right\rangle_{e}=\left\langle\mathrm{P}_{\partial} u-\widehat{u}_{h}, \psi \boldsymbol{\beta} \cdot \boldsymbol{n}\right\rangle_{\partial \Omega_{e} \backslash \Gamma^{-}},
$$

by our assumptions on the triangulation $\Omega_{h}$. Then

$$
\begin{aligned}
\left\langle\mathrm{P}_{\partial} u-\widehat{u}_{h}, \varphi\right\rangle_{e}= & \left\langle\mathrm{P}_{\partial} u-\widehat{u}_{h},\left(\psi-\mathbb{P}^{-} \psi\right) \boldsymbol{\beta} \cdot \boldsymbol{n}\right\rangle_{\partial \Omega_{e} \backslash \Gamma^{-}} \\
& +\left\langle\mathrm{P}_{\partial} u-\widehat{u}_{h}, \mathbb{P}^{-} \psi \boldsymbol{\beta} \cdot \boldsymbol{n}\right\rangle_{\partial \Omega_{e} \backslash \Gamma^{-}},
\end{aligned}
$$

and, by the error equation (2.9) with $v:=\mathbb{P}^{-} \psi$,

$$
\begin{aligned}
\left\langle\mathrm{P}_{\partial} u-\widehat{u}_{h}, \varphi\right\rangle_{e}= & \left\langle\mathrm{P}_{\partial} u-\widehat{u}_{h},\left(\psi-\mathbb{P}^{-} \psi\right) \boldsymbol{\beta} \cdot \boldsymbol{n}\right\rangle_{\partial \Omega_{e} \backslash \Gamma^{-}} \\
& +\left(\mathbb{P}^{+} u-u_{h}, \partial_{\boldsymbol{\beta}} \mathbb{P}^{-} \psi\right)_{\Omega_{e}} \\
& -\left(c\left(u-u_{h}\right), \mathbb{P}^{-} \psi\right)_{\Omega_{e}} \\
= & \left\langle\mathrm{P}_{\partial} u-\widehat{u}_{h},\left(\psi-\mathbb{P}^{-} \psi\right) \boldsymbol{\beta} \cdot \boldsymbol{n}\right\rangle_{\partial \Omega_{e} \backslash \Gamma^{-}} \\
& +\left(\mathbb{P}^{+} u-u_{h}, \partial_{\boldsymbol{\beta}} \mathbb{P}^{-} \psi\right)_{\Omega_{e}} \\
& -\left(c\left(\mathbb{P}^{+} u-u_{h}\right), \mathbb{P}^{-} \psi\right)_{\Omega_{e}} \\
& -\left(c\left(u-\mathbb{P}^{+} u\right), \mathbb{P}^{-} \psi\right)_{\Omega_{e}} .
\end{aligned}
$$


Hence, by the dual equation, (2.7a),

$$
\begin{aligned}
\left\langle\mathrm{P}_{\partial} u-\widehat{u}_{h}, \varphi\right\rangle_{e}= & \left\langle\mathrm{P}_{\partial} u-\widehat{u}_{h},\left(\psi-\mathbb{P}^{-} \psi\right) \boldsymbol{\beta} \cdot \boldsymbol{n}\right\rangle_{\partial \Omega_{e} \backslash \Gamma^{-}} \\
& +\left(\mathbb{P}^{+} u-u_{h}, \partial_{\boldsymbol{\beta}}\left(\mathbb{P}^{-} \psi-\psi\right)\right)_{\Omega_{e}} \\
& -\left(c\left(\mathbb{P}^{+} u-u_{h}\right), \mathbb{P}^{-} \psi-\psi\right)_{\Omega_{e}} \\
& -\left(c\left(u-\mathbb{P}^{+} u\right), \mathbb{P}^{-} \psi\right)_{\Omega_{e}} \\
= & : \sum_{i=1}^{4} T_{i} .
\end{aligned}
$$

Let us estimate the terms $T_{i}, i=1,2,3,4$. We begin by showing that $T_{1}+T_{2}=0$. After a simple integration by parts, we get that

$$
\begin{aligned}
T_{2} & =-\left\langle\mathbb{P}^{+} u-u_{h},\left(\psi-\mathbb{P}^{-} \psi\right) \boldsymbol{\beta} \cdot \boldsymbol{n}\right\rangle_{\partial \Omega_{e}}+\left(\partial_{\boldsymbol{\beta}}\left(\mathbb{P}^{+} u-u_{h}\right), \psi-\mathbb{P}^{-} \psi\right)_{\Omega_{e}} \\
& =-\left\langle\mathbb{P}^{+} u-u_{h},\left(\psi-\mathbb{P}^{-} \psi\right) \boldsymbol{\beta} \cdot \boldsymbol{n}\right\rangle_{\partial \Omega_{e}},
\end{aligned}
$$

by the orthogonality property of the projection $\mathbb{P}^{-},(2.3 \mathrm{a})$. Hence,

$$
\begin{aligned}
T_{1}+T_{2} & =\left\langle\mathrm{P}_{\partial} u-\widehat{u}_{h},\left(\psi-\mathbb{P}^{-} \psi\right) \boldsymbol{\beta} \cdot \boldsymbol{n}\right\rangle_{\partial \Omega_{e} \backslash \Gamma^{-}}-\left\langle\mathbb{P}^{+} u-u_{h},\left(\psi-\mathbb{P}^{-} \psi\right) \boldsymbol{\beta} \cdot \boldsymbol{n}\right)_{\partial \Omega_{e}} \\
& =0
\end{aligned}
$$

since, by the definition of $\mathbb{P}^{+}$and $\widehat{u}_{h}$, we have that $\mathrm{P}_{\partial} u=\mathbb{P}^{+} u$ and $\widehat{u}_{h}=u_{h}$ on any outflow face, and since, by the definition of $\mathbb{P}^{-}$, we have that

$$
\left\langle\omega,\left(\psi-\mathbb{P}^{-} \psi\right) \boldsymbol{\beta} \cdot \boldsymbol{n}\right\rangle_{\tilde{e}}=0 \quad \forall \omega \in \mathcal{P}^{k}(\tilde{e}),
$$

on any inflow face $\tilde{e}$.

It remains to estimate $T_{3}$ and $T_{4}$. The estimate of $T_{3}$ follows after a straightforward application of Cauchy-Schwarz inequality, the estimate of Theorem 2.2, the approximation property of the projection $\mathbb{P}^{-},(2.4)$, the stability estimate $(2.8)$. Indeed, we easily get that

$$
T_{3} \leq \mathrm{C}(c, s) h^{k+s+1}|u|_{H^{k+1}\left(\Omega_{e}\right)}\|\varphi\|_{H^{s}(e)},
$$

for $s \in[0, k+1]$. Let us estimate $T_{4}$. If we denote by $\mathrm{P}^{k-1}$ be the $L^{2}$-projection onto $V_{h}^{k-1}$, we have that

$$
\begin{aligned}
T_{4} & =\left(c\left(u-\mathbb{P}^{+} u\right), \psi-\mathbb{P}^{-} \psi\right)_{\Omega_{e}}-\left(u-\mathbb{P}^{+} u, c \psi\right)_{\Omega_{e}} \\
& =\left(c\left(u-\mathbb{P}^{+} u\right), \psi-\mathbb{P}^{-} \psi\right)_{\Omega_{e}}-\left(u-\mathbb{P}^{+} u, c \psi-\mathrm{P}^{k-1} c \psi\right)_{\Omega_{e}},
\end{aligned}
$$

by the orthogonality property of the projection $\mathbb{P}^{+},(2.3 \mathrm{a})$. Then we get

$$
T_{4} \leq \mathrm{C}(c, s) h^{k+s+1}\|\varphi\|_{H^{s}(e)}|u|_{H^{k+1}\left(\Omega_{e}\right)},
$$

for $s \in[0, k]$, by the approximation properties of the projections $\mathbb{P}^{+}, \mathbb{P}^{-}$, and $\mathrm{P}^{k-1}$, and the stability estimate (2.8). This completes the proof. 


\section{Extensions.}

3.1. The variable-degree DG method. The variable-degree DG approximation $u_{h}$ belongs to the finite dimensional space $V_{h}^{\boldsymbol{k}}$ which is given by

$$
V_{h}^{\boldsymbol{k}}=\left\{v \in L^{2}(\Omega),\left.v\right|_{K} \in \mathcal{P}^{k(K)}(K), \forall K \in \Omega_{h}\right\},
$$

where $\boldsymbol{k}=\left\{k(K), \forall K \in \Omega_{h}\right\}$.

The definition of $\mathbb{P}$ can easily be generalized for the variable-degree space. We define $\mathbb{P} u \in V_{h}^{\boldsymbol{k}}$ using the definition of the previous section but with the space $\mathcal{P}^{k-1}(K)$ replaced with $\mathcal{P}^{k(K)-1}(K)$ and the space $\mathcal{P}^{k}\left(e_{K}^{+}\right)$replaced with $\mathcal{P}^{k(K)}\left(e_{K}^{+}\right)$.

We need to impose a condition on the space $V_{h}^{\boldsymbol{k}}$ :

$$
k(K) \geq k\left(K^{\prime}\right) \text { whenever } e_{K}^{+} \subseteq e_{K^{\prime}}^{-}
$$

The following result can be proven in the same way as Theorem 2.2.

THEOREM 3.1. Let $u_{h} \in V_{h}^{\boldsymbol{k}}$ be the variable degree $D G$ approximation given by (2.1) with $V_{h}^{k}$ replaced with $V_{h}^{k}$. If $\Omega_{h}$ satisfies the flow conditions (1.3) with respect to $\beta$ and $V_{h}^{\boldsymbol{k}}$ satisfies the condition of the polynomial degrees (3.1), then the results of Theorem 2.2 hold for the variable-degree DG method.

3.2. Singularly Perturbed Problem. We present local error estimates of a DG method for the following singular perturbed problem in $\Omega \subset \mathbb{R}^{2}$

$$
\begin{array}{rlrl}
-\epsilon \triangle u+\boldsymbol{\beta} \cdot \nabla u+c u & =f & \text { in } \Omega, \\
u & =g & & \text { on } \partial \Omega .
\end{array}
$$

We use the interior penalty method to discretize the viscosity term $-\epsilon \triangle u$, see [2] and [9]. Any other DG method which is consistent and stable could be used to discretize this term, see [3].

Thus, the DG approximation $u_{h} \in V_{h}^{k}$, where $k \geq 1$, of (3.2) solves

$$
\epsilon A\left(u_{h}, v_{h}\right)+B\left(u_{h}, v_{h}\right)=F\left(v_{h}\right) \text { for all } v_{h} \in V_{h}^{k},
$$

where

$$
\begin{aligned}
A(\omega, v) & =(\nabla \omega, \nabla v)_{\Omega_{h}}-\sum_{e \in \mathcal{E}_{h}}\left(\left\langle\left\{\{\nabla \omega \rrbracket, \llbracket v \rrbracket\rangle_{e}+\left\langle\{\{\nabla v\}, \llbracket \omega \rrbracket\rangle_{e}-\frac{\eta}{h}\langle\llbracket \omega \rrbracket, \llbracket v \rrbracket\rangle_{e}\right),\right.\right.\right. \\
F(v) & =(f, v)_{\Omega_{h}}+\langle g, v \boldsymbol{\beta} \cdot \boldsymbol{n}\rangle_{\partial \Omega}+\epsilon\langle\nabla v \cdot \boldsymbol{n}, g\rangle_{\partial \Omega}-\epsilon \frac{\eta}{h}\langle g, v\rangle_{\partial \Omega} .
\end{aligned}
$$

The set $\mathcal{E}_{h}$ is the collection of edges of the triangulation $\Omega_{h}$. The parameter $\eta$ is large enough in order to ensure stability.

The average $\{\{\}$ and jump $\llbracket \cdot \rrbracket$ operators are defined as follows. For an interior edge $e$, we set

$$
\begin{array}{ll}
\{\boldsymbol{q}\}=\frac{1}{2}\left(\boldsymbol{q}_{1}+\boldsymbol{q}_{2}\right), & \llbracket \boldsymbol{q} \rrbracket=\boldsymbol{q}_{1} \cdot \boldsymbol{n}_{1}+\boldsymbol{q}_{2} \cdot \boldsymbol{n}_{2}, \\
\{\varphi\}=\frac{1}{2}\left(\varphi_{1}+\varphi_{2}\right), & \llbracket \varphi \rrbracket=\varphi_{1} \boldsymbol{n}_{1}+\varphi_{2} \boldsymbol{n}_{2},
\end{array}
$$

where $e=K_{1} \cap K_{2}, \boldsymbol{q}_{i}=\left.\boldsymbol{q}\right|_{K_{i}}, \varphi_{i}=\left.\varphi\right|_{K_{i}}$ and $\boldsymbol{n}_{i}$ is the exterior unit normal to $K_{i}, i=1,2$. For a boundary edge $e$, we set

$$
\{\{\boldsymbol{q}\}=\boldsymbol{q}, \llbracket \varphi \rrbracket=\varphi \boldsymbol{n}
$$


where $n$ is the outward unit normal. The quantities $\llbracket \boldsymbol{q} \rrbracket$ and $\{\{\varphi\}$ on boundary edges are not required, so they are left undefined.

In order to state the result we need to introduce a proper weight function. For simplicity we assume $\boldsymbol{\beta}=(1,0)$. Accordingly, we set, for fixed $x_{0}, y_{1}$ and $y_{2}$,

$$
\Omega_{0}=\left(\left(-\infty, x_{0}\right] \times\left[y_{1}, y_{2}\right]\right) \cap \Omega,
$$

and construct a function $\omega$ satisfying

$$
\begin{aligned}
C_{1} \leq \omega(x, y) & \leq C_{2}, & & \text { for }(x, y) \in \Omega_{0}, \\
|\omega(x, y)| & \leq C_{2} e^{-\left(x-x_{0}\right) / M \rho}, & & \text { for } x \geq x_{0}+h, \\
|\omega(x, y)| & \leq C_{2} e^{-\left(y-y_{2}\right) / M \sigma}, & & \text { for } y \geq y_{2}+h, \\
|\omega(x, y)| & \leq C_{2} e^{-\left(y_{1}-y\right) / M \sigma}, & & \text { for } y \leq y_{1}-h .
\end{aligned}
$$

Here $\rho \geq 0, \sigma \geq 0$ are parameters that will depend on the mesh size $h$ and $\epsilon$. We say that $\rho$ is the size of the upwind layer and $\sigma$ is the size of the crosswind layer. The positive constants $C_{1}, C_{2}$ and $M$ are fixed.

THEOREM 3.2. We assume $\epsilon \leq h$ and that our mesh is quasi-uniform. If $\Omega_{h}$ satisfies the flow conditions (1.3) with respect to $\boldsymbol{\beta}$. Then the error between $u_{h}$ given by (3.3) and u given by (3.2) is

$$
\begin{gathered}
\left\|\omega\left(u-u_{h}\right)\right\|_{L^{2}(\Omega)} \leq C\left(\left(1+\frac{\epsilon^{1 / 2}}{h}\right)\|\omega(u-\mathbb{P} u)\|_{L^{2}(\Omega)}+\epsilon^{1 / 2}\|\omega \nabla(u-\mathbb{P} u)\|_{L^{2}\left(\Omega_{h}\right)}\right. \\
\left.+h \epsilon^{1 / 2}\left\|\omega D^{2}(u-\mathbb{P} u)\right\|_{L^{2}\left(\Omega_{h}\right)}\right)
\end{gathered}
$$

where $\omega$ is given above with $\rho=\log \left(\frac{1}{h}\right) h, \sigma=h^{1 / 2}$ and $K$ is a sufficiently large fixed constant. Moreover, if $\Omega_{h}$ also satisfies the flow conditions (1.3) with respect to $-\boldsymbol{\beta}$, then we can choose $\sigma=\max \left(\epsilon^{1 / 2}, h\right)$.

We immediately get optimal weighted error estimates if $\epsilon \leq h^{2}$. The size of the crosswind layer $\sigma$ is typically $\sigma=h^{1 / 2}$ for general triangulations; see [10]. However, we see that the size of the crosswind layer is reduced to $\sigma=h$ if the flow conditions (1.3) for both $\boldsymbol{\beta}$ and $-\boldsymbol{\beta}$ are satisfied. This is exactly the result that was obtained for the streamline diffusion method in [14]. However, in [14] an almost uniform mesh condition was needed whereas for our results we only assume quasi-uniformity. Moreover, our results also hold for high-order elements.

The proof of Theorem 3.2 is very similar to the proof of the local estimates given in [10]. However, instead of using the $L^{2}$-projection one must use the projections $\mathbb{P}=\mathbb{P}^{+}$and $\mathbb{P}^{-}$used in this paper.

4. Concluding remarks. This paper contains the first instance in which the approximation error $\left\|u-u_{h}\right\|_{L^{2}\left(\Omega_{h}\right)}$, where $u$ is the solution of the transport-reaction equation and $u_{h}$ is given by the original DG method, is proven to be optimal in the mesh size $h$ and in the regularity of the exact solution. Unexpectedly, this happens with meshes whose main feature is to be, roughly speaking, aligned with the flow.

The fact that the direction of the flow $\boldsymbol{\beta}$ is a constant and the use of simplexes to define the triangulations $\Omega_{h}$ seem to play a major role in the result. The case of variable $\boldsymbol{\beta}$ and simplexes with curved boundaries is the subject of ongoing research.

Appendix: The construction of triangulations satisfying the flow conditions. Let us show that it is always possible to construct a triangulation of the domain $\Omega \subset \mathbb{R}^{d}$ satisfying the flow conditions (1.3). 


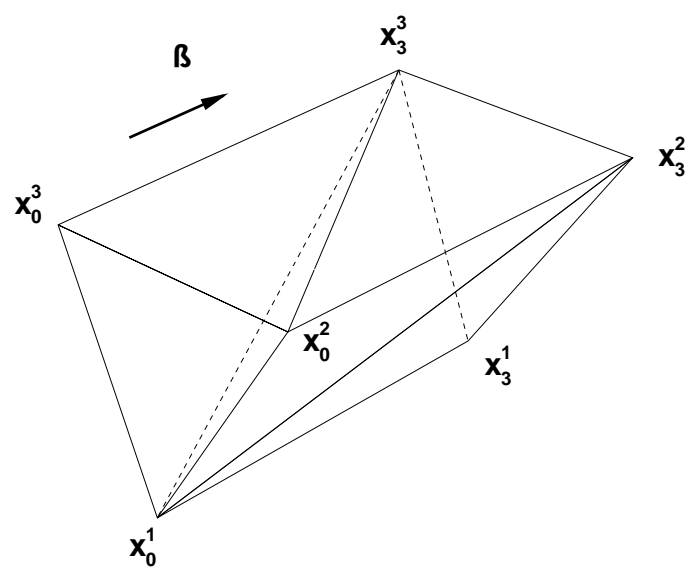

FIG. 4.1. Detail of the construction of a 3D triangulation satisfying the flow conditions.

We can do this as follows. First, we triangulate the inflow border of $\Omega, \Gamma^{-}$, by using $(d-1)$-dimensional simplexes $T$; let $\Gamma_{h}^{-}:=\{T\}$ be such a triangulation. Next, for each $T$, we construct the $d$-dimensional prism

$$
\mathcal{T}_{T}:=(T \oplus\{\lambda \boldsymbol{\beta}: \lambda \in \mathbb{R}\}) \cap \Omega,
$$

which we are going to triangulate by using conforming $d$-dimensional simplexes. Note that the triangulations of the prisms are completely independent of each other since no conformity between them is required thanks to the flow conditions 1.3.

To triangulate the prism $\mathcal{T}_{T}$, we only have to show that, if $T$ and $T^{\prime}$ are two $(d-1)$ dimensional simplexes whose vertices are on the border of $\mathcal{T}_{T}$, we can triangulate the convex hull of $T$ and $T^{\prime}, C H\left(T, T^{\prime}\right)$, by using exactly $d d$-dimensional simplexes each of which has one inflow and one outflow face. Those simplexes are $C H\left(T_{i-1}, T_{i}\right)$, $i=1, \ldots, d$, where the $(d-1)$-dimensional simplexes $T_{i}, i=0, \ldots, d$, are constructed in such a way that $T_{0}:=T, T_{d}:=T^{\prime}$, and, assuming that $T$ is an inflow face, that $T_{i-1}$ is the only inflow face of the simplex $C H\left(T_{i-1}, T_{i}\right)$ and $T_{i}$ is the only outflow face of the simplex $C H\left(T_{i-1}, T_{i}\right)$.

If we identify the $(d-1)$-dimensional simplex $T_{i}$ with its set of vertices, $\left\{\boldsymbol{x}_{i}^{j}\right\}_{j=1}^{d}$, it is not difficult to see that we can take

$$
\begin{aligned}
& T_{0} \equiv\left\{\boldsymbol{x}_{0}^{1}, \boldsymbol{x}_{0}^{2}, \ldots, \boldsymbol{x}_{0}^{d-2}, \boldsymbol{x}_{0}^{d-1}, \boldsymbol{x}_{0}^{d}\right\}, \\
& T_{1} \equiv\left\{\boldsymbol{x}_{0}^{1}, \boldsymbol{x}_{0}^{2}, \ldots, \boldsymbol{x}_{0}^{d-2}, \boldsymbol{x}_{0}^{d-1}, \boldsymbol{x}_{d}^{d}\right\}, \\
& T_{2} \equiv\left\{\boldsymbol{x}_{0}^{1}, \boldsymbol{x}_{0}^{2}, \ldots, \boldsymbol{x}_{0}^{d-2}, \boldsymbol{x}_{d}^{d-1}, \boldsymbol{x}_{d}^{d}\right\}, \\
& T_{3} \equiv\left\{\boldsymbol{x}_{0}^{1}, \boldsymbol{x}_{0}^{2}, \ldots, \boldsymbol{x}_{d}^{d-2}, \boldsymbol{x}_{d}^{d-1}, \boldsymbol{x}_{d}^{d}\right\}, \\
& T_{d} \equiv\left\{\boldsymbol{x}_{d}^{1}, \boldsymbol{x}_{d}^{2}, \ldots, \boldsymbol{x}_{d}^{d-2}, \boldsymbol{x}_{d}^{d-1}, \boldsymbol{x}_{d}^{d}\right\},
\end{aligned}
$$

respectively. An example of this construction is illustrated in the Fig. 4.1. 


\section{REFERENCES}

[1] S. Adjerid and T.C. Massey, A posteriori discontinuous finite element error estimation for two-dimensional hyperbolic problems, Comput. Methods Appl. Mech. Engrg. 191 (2002), $5877-5897$.

[2] D.N. Arnold, An interior penalty finite element method with discontinuous elements, SIAM J. Numer. Anal., 19 (1982), 742-760.

[3] D. N. Arnold, F. Brezzi, B. Cockburn, and L. D. Marini, Unified analysis of discontinuous Galerkin methods for elliptic problems, SIAM J. Numer. Anal. 39 (2002), 1749-1779.

[4] P. Bastian and B. Rivière, Superconvergence and $H$ (div) projection for discontinuous Galerkin methods, Internat. J. Numer. Methods Fluids 42 (2003), 1043-1057.

[5] F. Brezzi and M. Fortin, Mixed and hybrid finite element methods, Springer Verlag, 1991.

[6] B. Cockburn, B. Dong and J. Guzmán, A superconvergent LDG-hybridizable Galerkin method for second-order elliptic problems, Submitted to Math. Comp.

[7] B. Cockburn, G. Kanschat, and D. Schötzau, A locally conservative LDG method for the incompressible Navier-Stokes equations, Math. Comp. 74 (2005), 1067-1095.

[8] B. Cockburn, D. Schötzau, and J. Wang, Discontinuous Galerkin methods for incompressible elastic materials, Comput. Methods Appl. Mech. Engng. 195 (2006), 3184-3204, C. Dawson, Ed.

[9] J. Gopalakrishnan, G. Kanschat, A multilevel discontinuous Galerkin method, Numer. Math. 95 (2003), 527-550.

[10] J. Guzmán, Local analysis of discontinuous Galerkin methods applied to singularly perturbed problems, J. Numer. Math. 14 (2006), 41-56.

[11] C. Johnson and J. Pitkäranta, An analysis of the discontinuous Galerkin method for a scalar hyperbolic equation, Math. Comp., 46 (1986), 1-26.

[12] P. Lesaint and P.-A Raviart, On a finite element method for solving the neutron transport equation, In: Mathematical Aspects for Finite Elements in Partial Differential Equations (Ed. C. de Boor), Academic Press, New York, 1974.

[13] T.E. Peterson, A note on the convergence of the discontinuous Galerkin method for a scalar hyperbolic equation, SIAM J. Num. Anal., 28 (1991) , 133-140.

[14] G. Zhou and R. Rannacher, Pointwise superconvergence of the streamline diffusion finiteelement method, Numer. Methods Partial Differential Equations, 12 (1996), 123-145

[15] W. Reed, T. Hill, Triangular mesh methods for the neutron transport equation, LA-UR-73-479, 1973.

[16] G.R. Richter, An optimal-order error estimate for the discontinuous Galerkin method. Math. Comp. 50 (1988), 75-88. 